Bulletin of the Section of Logic

Volume 45/2 (2016), pp. 93-109

http://dx.doi.org/10.18778/0138-0680.45.2.03

Gemma Robles, Sandra M. López, José M. Blanco, and Marcos M. Recio and Jesús R. Paradela

\title{
A 2-SET-UP ROUTLEY-MEYER SEMANTICS FOR THE 4-VALUED RELEVANT LOGIC E4
}

\begin{abstract}
The logic BN4 can be considered as the 4-valued logic of the relevant conditional and the logic E4, as the 4-valued logic of (relevant) entailment. The aim of this paper is to endow E4 with a 2-set-up Routley-Meyer semantics. It is proved that E4 is strongly sound and complete w.r.t. this semantics.

Keywords: Relevant logics; many-valued logics; 4-valued logics; 2-set-up Routley-Meyer semantics.
\end{abstract}

\section{Introduction}

Concerning the 2-set-up Routley-Meyer semantics he defined for the logic RM3, Brady notes "the method of proof is fairly general and it is hoped that other model structures can be axiomatized by appropriate modifications to the proof" ([2], p. 9). The aim of this paper is to provide one of such model structures: we endow the 4-valued logic of entailment E4 with a 2-set-up Routley-Meyer semantics.

Brady's logic BN4 can be viewed as the 4-valued logic of the relevant conditional while the logic E4, introduced in [5], can be regarded as the 4 -valued logic of (relevant) entailment. The logic BN4 was defined by Brady in [2] and it can intuitively be described as a 4-valued extension of contractionless relevant logic RW (that is, relevant logic $\mathrm{R}$ minus the contraction axiom, i.e., $[A \rightarrow(A \rightarrow B)] \rightarrow(A \rightarrow B))$. On its part, E4 can intuitively be described as a 4 -valued extension of the logic of entailment 
E minus the reductio axiom, i.e., $(A \rightarrow \neg A) \rightarrow \neg A$. Thus, E4 is related to BN4 in a similar way to which Anderson and Belnap's logic of entailment $\mathrm{E}$ is related to their logic of relevant conditional R (cf. [1] about these logics).

According to Meyer et al., "BN4 is the correct logic for the 4-valued situation where the extra values are to be interpreted in the both and neither senses" ([4], p. 25). On the other hand, E4 can be viewed as the "4-valued logic of relevant entailment" on the basis of the two following facts: (1) E4 enjoys the "weak relevance property" that is characteristic of such logics as R-mingle. This property reads as follows: if $A \rightarrow B$ is a theorem, then either $A$ and $B$ share at least a propositional variable or both $\neg A$ and $B$ are theorems (cf. [1], p. 417); (2) as it is the case with the logic of entailment $\mathrm{E}$ (cf. [1], $\S 4.3$ and references therein), E4 encloses "a theory of logical necessity" when the necessity operator is defined via the conditional as follows: $\square A=_{d f}(A \rightarrow A) \rightarrow A$ (cf. section 5 below).

In [2], BN4 is endowed with both a bivalent Belnap-Dunn type semantics and a 2-set-up Routley-Meyer ternary relational semantics. On the other hand, in [5], a bivalent Belnap-Dunn type semantics is defined for the logic E4, which, nevertheless, still lacks the latter kind of semantics mentioned above. The aim of this paper is then to provide a 2-set-up Routley-Meyer ternary relational semantics for E4.

The paper is organized as follows. In section 2, the logic E4 is defined and some of its theorems to be used in the completeness proofs are remarked. In section 3, the 2-set-up semantics is characterized and the soundness theorem is proved. In section 4, E4 is proved complete w.r.t. the semantics defined in the previous section. Finally, in section 5, the necessity operator referred to above is introduced in the models by a binary accessibility relation in the customary way.

\section{The logic E4}

Firstly, we define the logical language and the notion of logic used in the paper.

DeFinition 2.1 (Languages). The propositional language consists of a denumerable set of propositional variables $p_{0}, p_{1}, \ldots, p_{n}, .$. , and the following connectives $\rightarrow$ (conditional), $\wedge$ (conjunction), $\vee$ (disjunction), $\neg$ (nega- 
tion). The biconditional $(\leftrightarrow)$ and the set of wffs are defined in the customary way. $A, B$ etc. are metalinguistic variables.

Definition 2.2 (Logics). A logic $S$ is a structure $\left(L, \vdash_{S}\right)$ where $L$ is a propositional language and $\vdash_{S}$ is a (proof-theoretical) consequence relation defined on $L$ by a set of axioms and a set of rules of derivation. The notions of 'proof' and 'theorem' are understood as it is customary in Hilbert-style axiomatic systems $\left(\Gamma \vdash_{S} A\right.$ means that $A$ is derivable from the set of $w f f s$ $\Gamma$ in $S$; and $\vdash_{S} A$ means that $A$ is a theorem of $S$ ).

Next, the logic E4 is defined (cf. [5]):

Definition 2.3. The logic E4 is axiomatized with the following axioms and rules of inference.

Axioms

$$
\begin{aligned}
& \text { A1. } A \rightarrow A \\
& \text { A2. }(A \wedge B) \rightarrow A /(A \wedge B) \rightarrow B \\
& \text { A3. }[(A \rightarrow B) \wedge(A \rightarrow C)] \rightarrow[A \rightarrow(B \wedge C)] \\
& \text { A4. } A \rightarrow(A \vee B) / B \rightarrow(A \vee B) \\
& \text { A5. }[(A \rightarrow C) \wedge(B \rightarrow C)] \rightarrow[(A \vee B) \rightarrow C] \\
& \text { A6. }[A \wedge(B \vee C)] \rightarrow[(A \wedge B) \vee(A \wedge C)] \\
& \text { A7. }(A \rightarrow B) \rightarrow[(B \rightarrow C) \rightarrow(A \rightarrow C)] \\
& \text { A8. }[A \rightarrow(A \rightarrow B)] \rightarrow(A \rightarrow B) \\
& \text { A9. }[[(A \rightarrow A) \wedge(B \rightarrow B)] \rightarrow C] \rightarrow C \\
& \text { A10. }(A \rightarrow \neg B) \rightarrow(B \rightarrow \neg A) \\
& \text { A11. }(\neg A \rightarrow B) \rightarrow(\neg B \rightarrow A) \\
& \text { A12. }[(A \rightarrow B) \wedge(A \wedge \neg B)] \rightarrow \neg(A \rightarrow B) \\
& \text { A13. }(A \rightarrow B) \vee \neg(A \rightarrow B) \\
& \text { A14. }[\neg(A \rightarrow B) \wedge(\neg A \wedge B)] \rightarrow(A \rightarrow B) \\
& \text { A15. } \neg A \rightarrow[A \vee(A \rightarrow B)]
\end{aligned}
$$

Rules of derivation

Adjunction (Adj): $A \& B \Rightarrow A \wedge B$ Modus Ponens (MP): $A \& A \rightarrow B \Rightarrow B$ 
We note the following remarks.

REMARK 2.4 (The logic of entailmet $\mathrm{E}$ minus the reductio axiom). $W e$ note that the logic of entailment $E$ minus the reductio axiom (i.e., $(A \rightarrow$ $\neg A) \rightarrow \neg A)$ can be axiomatized with A1-A11, Adj and MP. Notice, on the other hand, that although A12 and A13 are theorems of E, A14 and A15 are neither theorems of $E$ nor of $R$.

REMARK 2.5 (The logic BN4). Brady's logic BN4 is axiomatized with the following axioms: A1-A7, A10, A11 and A15 in Definition 2.3, and, in addition: $A \rightarrow[(A \rightarrow B) \rightarrow B],(\neg A \wedge B) \rightarrow(A \rightarrow B),(A \vee \neg B) \vee(A \rightarrow B)$ and $A \vee[\neg(A \rightarrow B) \rightarrow A]$. The rules are $A d j, M P$ and Disjunctive Modus Ponens: $C \vee(A \rightarrow B) \& C \vee A \Rightarrow C \vee B$ (cf. [2] and [5]). Notice that contractionless relevant logic $R, R W$, can be axiomatized with A1-A\%, A10, $A 11, A \rightarrow[(A \rightarrow B) \rightarrow B]$, Adj and $M P$.

Next, we introduce the matrices MBN4 and ME4 upon which BN4 and E4 are defined, respectively (cf. [2] and [5]).

DeFinition 2.6 (The matrix MBN4). The propositional language consists of the connectives $\rightarrow, \wedge, \vee$ and $\neg$. The matrix $M B N 4$ is the structure $(\mathcal{V}, D$, F) where (1) $\mathcal{V}$ is $\{0,1,2,3\}$; (2) $D=\{3,2\}$; (3) $\mathrm{F}=\left\{f_{\rightarrow}, f_{\wedge}, f_{\vee}, f_{\neg}\right\}$ where $f_{\rightarrow}, f_{\wedge}, f_{\vee}, f_{\neg}$ are defined according to the following truth-tables.

\begin{tabular}{|c|c|c|c|c|c|c|c|c|c|c|c|c|c|c|c|c|}
\hline$\rightarrow$ & 0 & 1 & 2 & 3 & $\wedge$ & 0 & 1 & 2 & 3 & V & 0 & 1 & 2 & 3 & & $\neg$ \\
\hline 0 & 3 & 3 & 3 & 3 & 0 & 0 & 0 & 0 & 0 & 0 & 0 & 1 & 2 & 3 & 0 & 3 \\
\hline 1 & 1 & 3 & 1 & 3 & 1 & 0 & 1 & 0 & 1 & 1 & 1 & 1 & 3 & 3 & 1 & 1 \\
\hline 2 & 0 & 1 & 2 & 3 & 2 & 0 & 0 & 2 & 2 & 2 & 2 & 3 & 2 & 3 & 2 & 2 \\
\hline 3 & 0 & 1 & 0 & 3 & 3 & 0 & 1 & 2 & 3 & 3 & 3 & 3 & 3 & 3 & 3 & 0 \\
\hline
\end{tabular}

DeFinition 2.7 (The matrix E4). The propositional language is the same as in $M B N_{4}$. The matrix $E_{4}$ is the structure $(\mathcal{V}, D, F)$ where $\mathcal{V}, D$ and $\mathrm{F}$ are defined exactly as in MBN4 except for $f \rightarrow$ which is defined according to the following truth-table:

\begin{tabular}{l|llll}
$\rightarrow$ & 0 & 1 & 2 & 3 \\
\hline 0 & 3 & 3 & 3 & 3 \\
1 & 0 & 2 & 0 & 3 \\
2 & 0 & 0 & 2 & 3 \\
3 & 0 & 0 & 0 & 3
\end{tabular}


In [5] (resp. [2]) it is proved that the logic E4 (resp. BN4) is determined by the matrix ME4 (resp. MBN4). We end the section by remarking some theorems that will be useful in the completeness proof.

Proposition 2.8 (Some theorems of E4). The following are provable in E4

$$
\begin{aligned}
& \text { T1. }[A \wedge(A \rightarrow B)] \rightarrow B \\
& \text { T2. } A \rightarrow \neg \neg A \\
& \text { T3. } \neg \neg A \rightarrow A \\
& \text { T4. } \neg(A \vee B) \leftrightarrow(\neg A \wedge \neg B) \\
& \text { T5. } \neg(A \wedge B) \leftrightarrow(\neg A \vee \neg B) \\
& \text { T6. }(A \rightarrow B) \rightarrow(\neg B \rightarrow \neg A) \\
& \text { T7. } \neg B \rightarrow[\neg A \vee(\neg A \rightarrow B)] \\
& \text { T8. } A \rightarrow[B \vee \neg(A \rightarrow B)] \\
& \text { T9. }[\neg(A \rightarrow B) \wedge \neg A] \rightarrow A \\
& \text { T10. }(A \vee \neg B) \vee(A \rightarrow B) \\
& \text { T11. }[\neg(A \rightarrow B) \wedge B] \rightarrow \neg B
\end{aligned}
$$

Proof: T1-T11 are verified by matrix ME4 (in case a tester is needed, the reader can use that in [3]).

\section{A 2-set-up Routley-Meyer semantics for E4}

In this section, E4 is endowed with a 2-set-up Routley-Meyer semantics (RM-semantics). We begin by defining the concept of a model and related notions.

DeFInITION 3.1 (2-set-up E4-models). Let $*$ be an involutive unary operation defined on the set $K$. That is, for any $x \in K, x=x^{* *}$, and let $K$ be a set that contains an element (labelled 0$)$ as well as $0^{*}$ and no other elements. A 2-set-up E4-model (E4-model, for short) is a structure $(K, *, R, \models)$ where $R$ is a ternary relation on $K$ defined as follows: if $a, b, c \in K$, then Rabc iff $a=0^{*}$ or $b=c$. And $\vDash$ is a (valuation) relation from $K$ to the set of all wffs such that the following conditions (clauses) are satisfied for every propositional variable $p$, wffs $A, B$ and $a \in K$ : 
(i). $a \vDash p$ or $a \not \models p$

(ii). $a \vDash A \wedge B$ iff $a \vDash A$ and $a \vDash B$

(iii). $a \vDash A \vee B$ iff $a \vDash A$ or $a \vDash B$

(iv). $a \vDash A \rightarrow B$ iff for all $b, c \in K,($ Rabc and $b \vDash A) \Rightarrow c \vDash B$

(v). $a \vDash \neg A$ iff $a^{*} \not \models A$

Next, the notions of truth in an E4-model, validity and semantic consequence are defined.

Definition 3.2 (Truth in a E4-model). A wff $A$ is true in a E4-model iff $0 \vDash A$ in this model.

Definition 3.3 (E4-validity). A wff $A$ is E4-valid (in symbols, $\vDash_{E 4} A$ ) iff $0 \vDash A$ in all E4-models.

Definition 3.4 (Semantic E4-consequence). For any set of wffs $\Gamma$ and wff $A, \Gamma \vDash_{M} A$ ( $A$ is a consequence of $\Gamma$ in the E4-model $\left.M\right)$ iff $0 \vDash A$ if $0 \vDash \Gamma$ $(0 \vDash \Gamma$ iff $0 \vDash B$ for all $B \in \Gamma)$. Then, $\Gamma \vDash_{E 4} A$ ( $A$ is an E4-consequence of $\Gamma$ ) iff $\Gamma \vDash_{M}$ A for each $E$ 4-model $M$.

We note a couple of remarks and then prove a proposition and lemma useful in the proof of soundness.

REMARK 3.5 ( $R$ in RM3 and in BN4). We note that Brady (cf. [2], p. 18 and $p$. 29) defines the ternary relation $R$ in the 2-set-up Routley-Meyer semantics for RM3 and BN4 as follows. For all $a, b, c \in K:$ Rabc iff $a \neq 0$ or $b \neq 0$ or $c=0$, in RM3; Rabc iff $(a \neq 0$ or $b=c)$ \& $\left[a \neq 0^{*}\right.$ or $\left(b=0\right.$ \& $\left.\left.c=0^{*}\right)\right]$ in BN4. In other words, and supposing $0 \neq 0^{*}$, $R$ is the total ternary relation minus $R 000^{*}$ in $R M 3$; and in $B N 4, R$ is $\left\{R 000, R 00^{*} 0^{*}, R 0^{*} 00^{*}\right\}$.

REMARK 3.6 (Ternary relations in $K$ ). Suppose $0 \neq 0^{*}$. Then, from the definition of $R$ (Definition 3.1), it follows that, for any E4-model $M$, the following ternary relations are the only ones holding in $M$ : (a) R000; (b) $R 0^{*} 0^{*} 0^{*}$; (c) $R 0^{*} 0^{*} 0$; (d) $R 0^{*} 00^{*}$, (e) $R 00^{*} 0^{*}$ and (f) $R 0^{*} 00$. (Or, put in other words, $R$ is the total ternary relation minus $R 000^{*}$ and $R 00^{*} 0$.) 
By Definition 3.1, we have $0 \vDash \neg A$ iff $0^{*} \not \models A$. But we have also the following proposition.

Proposition $3.7\left(0^{*} \vDash \neg A\right.$ iff $\left.0 \not \models A\right)$. For any E4-model $M$ and wff $A$, $0^{*} \vDash \neg A$ iff $0 \not \models A$.

Proof: Immediate by clause $\mathrm{v}$ in Definition 3.1 and the involutiveness of $*: 0^{*} \vDash \neg A$ iff (clause v) $0^{* *} \not \models A$ iff ( $*$ is involutive) $0 \not \models A$.

Lemma 3.8 (Entailment lemma). For any wffs $A, B, \vDash_{E_{4}} A \rightarrow B$ iff $(a \vDash A \Rightarrow a \vDash B$, for all $a \in K)$ in all E4-models.

Proof: (I) Suppose (1) $\vDash_{\mathrm{E} 4} A \rightarrow B$ and (2) $0 \vDash A$ (resp., $0^{*} \vDash A$ ). By Remark 3.6, (3) $R 000$ (resp., $R 00^{*} 0^{*}$ ). So, (4) $0 \vDash B$ (resp., $0^{*} \vDash B$ ) by applying clause iv (Definition 3.1) to 1, 2 and 3. (II) Suppose (1) $a \vDash A \Rightarrow$ $a \vDash B$ for all $a \in K$ in any E4-model. Further, suppose (2) $R 0 x y$ and $x \vDash A$ for $x, y \in K$. We have to consider only two cases, viz., $R 000$ and $R 00^{*} 0^{*}$ (cf. Remark 3.6). But by clause iv (Definition 3.1), it is clear that $\models_{\mathrm{E} 4} A \rightarrow B$ iff $(R 000 \& \quad \& \vDash A) \Rightarrow 0 \vDash B$ and $\left(R 00^{*} 0^{*} \quad \& \quad 0^{*} \vDash A\right) \Rightarrow 0^{*} \vDash B$. Thus, given $R 000$ and $0 \vDash A$ (resp., $R 00^{*} 0^{*}$ and $0^{*} \vDash A$ ), and the hypothesis 1 , we have $0 \vDash B$ (resp., $0^{*} \vDash B$ ), as it was required.

Theorem 3.9 (Soundness of E4). For any set of wffs $\Gamma$ and wff $A$, if $\Gamma \vdash_{E 4} A$, then $\Gamma \vDash_{E_{4}} A$.

Proof: (I) $A \in \Gamma$. The proof is trivial.

(II) The rules preserve E4-validity. If $A$ has been derived by Adj, the proof is trivial. So, let us consider the case when $A$ has been derived by MP. Suppose then $\Gamma \vDash_{\mathrm{E} 4} B \rightarrow A, \Gamma \vDash_{\mathrm{E} 4} B$ for some wff $B$. Further, suppose $0 \vDash \Gamma$. Then, (1) $0 \vDash B \rightarrow A$ and $0 \vDash B$. By Remark 3.6, (2) R000. So, $0 \vDash A$ follows by applying clause iv to 1 and 2 .

(III) The axioms A1-A15 are E4-valid. A few instances will suffice as an illustration. We prove the E4-validity of A12-A15, the lesser known axioms in relevant logics (A1-A11 are proved similarly; actually, similarly as they are proved in standard general RM-semantics). The use of the Entailment lemma will simplify the proofs.

$A 15 \neg A \rightarrow[A \vee(A \rightarrow B)]$ is E4-valid. We have to take into consideration the following two cases. For an arbitrary E4-model, (i) $0 \vDash \neg A \Rightarrow 0 \vDash$ 
$A \vee(A \rightarrow B)$ and (ii) $0^{*} \vDash \neg A \Rightarrow 0^{*} \vDash A \vee(A \rightarrow B)$. Case (i): Suppose (1) $0 \vDash \neg A$ (i.e., $0^{*} \not \models A$ ) but $(2) 0 \not \models A \vee(A \rightarrow B$ ), i.e., $0 \not \models A$ and $0 \not \models A \rightarrow B$. By clause iv, we have (3) $R 0 x y, x \vDash A$ and $y \not \models B$ for $x, y \in K$. Then, either (4) $R 000$ or (5) $R 00^{*} 0^{*}$ (cf. Remark 3.6). Suppose 4 . Then $0 \vDash A$ follows by 3 , contradicting 2 . Now, suppose 5 . Then, by $3,0^{*} \vDash A$, contradicting 1. Case (ii): Suppose (1) $0^{*} \vDash \neg A$ (i.e., $0 \not \models A$ ) but (2) $0^{*} \not \models A \vee(A \rightarrow B)$, i.e., $0^{*} \not \models A$ and $0^{*} \not \models A \rightarrow B$. By clause iv, we have (3) $R 0^{*} x y, x \vDash A$ and $y \not \models B$ for $x, y \in K$. We have to consider the four following alternatives: (4) $R 0^{*} 0^{*} 0^{*}$, (5) $R 0^{*} 0^{*} 0$; (6) $R 0^{*} 00^{*}$, (7) $R 0^{*} 00$. But by using 3 similarly as in case i, 4 and 5 are impossible by 2 , and 6 and 7 , by 1 .

$A 14[\neg(A \rightarrow B) \wedge(\neg A \wedge B)] \rightarrow(A \rightarrow B)$ is E4-valid. We prove cases (i) and (ii) for an arbitrary E4-model. Case (i): $0 \vDash \neg(A \rightarrow B) \wedge(\neg A \wedge B) \Rightarrow$ $0 \vDash A \rightarrow B$. Suppose (1) $0 \vDash \neg\left(A \rightarrow B\right.$ ) (i.e., $0^{*} \not \models A \rightarrow B$ ), $0 \vDash \neg A$ (i.e., $\left.0^{*} \not \models A\right), 0 \vDash B$ but (2) $0 \not \models A \rightarrow B$ for wffs $A, B$. By 2, we have (3) $R 0 x y$, $x \vDash A$ and $y \not \models B$ for $x, y \in K$. Then, either (4) $R 000$ or (5) $R 00^{*} 0^{*}$. But both 4 and 5 are impossible by using 1 and 3 similarly as in the proof of the E4-validity of A15. Case (ii): $0^{*} \vDash \neg(A \rightarrow B) \wedge(\neg A \wedge B) \Rightarrow 0^{*} \vDash A \rightarrow B$. Suppose (1) $0^{*} \vDash \neg(A \rightarrow B$ ) (i.e., $0 \not \models A \rightarrow B), 0^{*} \vDash \neg A$ (i.e., $0 \not \models A$ ) and $0^{*} \vDash B$ but $(2) 0^{*} \not \models A \rightarrow B$. By $1(0 \not \models A \rightarrow B)$, we have $R 0 x y, x \vDash A$ and $y \not \models B$ for $x, y \in K$, whence (3) $R 000,0 \vDash A$ and $0 \not \models B$ or (4) $R 00^{*} 0^{*}$, $0^{*} \vDash A$ and $0^{*} \not \models B$. But 3 and 4 are impossible by $1\left(0 \not \models A\right.$ and $\left.0^{*} \vDash B\right)$.

The E4-validity of A12 is proved similarly as in the case of A14. So, we conclude the proof of the soundness theorem by proving A13 E4-valid.

$A 13(A \rightarrow B) \vee \neg(A \rightarrow B)$ is E4-valid. Suppose for an E4-model, (1) $0 \not \models(A \rightarrow B) \vee \neg(A \rightarrow B)$. Then, (2) $0 \not \models A \rightarrow B$ and (3) $0 \not \models \neg(A \rightarrow B)$ (i.e., $0^{*} \vDash A \rightarrow B$ ). By 2 , we have (4) $R 0 x y, x \vDash A$ and $y \not \models B$ for some $x, y \in K$. Now, either (5) $R 000,0 \vDash A$ and $0 \not \models B$ or (6) $R 00^{*} 0^{*}, 0^{*} \vDash A$ and $0^{*} \not \models B$. Suppose 5. We have (7) $R 0^{*} 00$ (cf. Remark 3.6). Then, $0 \vDash B$ follows (contradicting 5 ) by applying clause iv to $3\left(0^{*} \vDash A \rightarrow B\right)$, $5(0 \vDash A)$ and 7 . Suppose 6 , on the other hand. We have (8) $R 0^{*} 0^{*} 0^{*}$ (cf. Remark 3.6). Then, $0^{*} \vDash B$ follows (contradicting 6 ) by applying clause iv to $3\left(0^{*} \vDash A \rightarrow B\right), 6\left(0^{*} \vDash A\right)$ and 8 .

Any E4-model in which $0=0^{*}$ verifies, of course, all classical twovalued propositional tautologies. But this is not necessarily so in models where $0 \neq 0^{*}$. Let us propose some examples. (The connectives of E4 are matched in the obvious way with the connectives of classical propositional logic.) 
$A \vee \neg A$ is not E4-valid. Consider an E4-model in which $0 \neq 0^{*}$ and for a propositional variable $p_{k}, 0 \not \models p_{k}$ and $0^{*} \vDash p_{k}$. Then, $0 \not \models \neg p_{k}$ and thus, $0 \not \models p_{k} \vee \neg p_{k}$.

$A \vee(A \rightarrow B)$ is not E4-valid. Consider an E4-model in which $0 \neq 0^{*}$ and for propositional variables $p_{i}, p_{k}(i \neq k)$ we have $0 \not \models p_{i}, 0^{*} \vDash p_{i}$ and $0^{*} \not \models p_{k}$ (it is indifferent how $p_{k}$ is evaluated in 0 ). Given $R 00^{*} 0^{*}, 0 \not \models p_{i} \rightarrow p_{k}$. Thus, $0 \not \models p_{i} \vee\left(p_{i} \rightarrow p_{k}\right)$.

$A \rightarrow(B \rightarrow A)$ is not E4-valid. Consider an E4-model in which $0 \neq 0^{*}$ and for propositional variables $p_{i}, p_{k}(i \neq k)$ we have $0 \vDash p_{i}, 0^{*} \not \models p_{i}, 0^{*} \vDash p_{k}$ (it is indifferent how $p_{k}$ is evaluated in 0 ). Given $R 00^{*} 0^{*}, 0 \not \models p_{k} \rightarrow p_{i}$. Thus, $0 \not \models p_{i} \rightarrow\left(p_{k} \rightarrow p_{i}\right)$ (cf. Lemma 3.8).

$A \rightarrow[(A \rightarrow B) \rightarrow B]$ is not E4-valid. Consider an E4-model in which $0 \neq 0^{*}$ and for propositional variables $p_{i}, p_{k}(i \neq k)$ we have $0 \not \models p_{i}, 0^{*} \vDash p_{i}$, $0 \not \models p_{k}$ and $0^{*} \vDash p_{k}$. Given $R 000, R 00^{*} 0^{*}, 0 \not \models p_{i}$ and $0^{*} \vDash p_{k}$, we have (1) $0 \vDash p_{i} \rightarrow p_{k}$. By $R 0^{*} 00$ and $0 \not \models p_{k},(2) 0^{*} \not \models\left(p_{i} \rightarrow p_{k}\right) \rightarrow p_{k}$. By $2,0^{*} \vDash p_{i}$ and the Entailment lemma (Lemma 3.8), $0 \not \models p_{i} \rightarrow\left[\left(p_{i} \rightarrow p_{k}\right) \rightarrow p_{k}\right]$.

In view of these examples it will be natural to ask why not to require $0 \neq$ $0^{*}$ in Definition 3.1. The answer is that in the canonical model construction defined in the next section, the crucial regular prime theory $\mathcal{T}$ and its *-image $\mathcal{T}^{*}$ do not seem to be proved equivalent and non-equivalent either.

\section{Completeness of E4}

We prove the strong completeness of $\mathrm{E} 4$ w.r.t. the semantics defined in the previous section by using a canonical model construction. We proceed as follows. Let $\Gamma$ be a set of wffs and $A$ a wff such that $\Gamma \nvdash_{\mathrm{E} 4} A$. Then, we show that there is a regular prime theory $\mathcal{T}$ (the notions are defined below) such that $\Gamma \subseteq \mathcal{T}$ and $A \notin \mathcal{T}$. This means that $A$ is not a consequence of $\Gamma$ from a canonical point of view to be defined, whence $\Gamma \nvdash_{\mathrm{E} 4} A$ follows. We begin by defining the notion of a theory and the classes of theories of interest in the present paper.

Definition 4.1 (E4-theories). An E4-theory (theory, for short) is a set of formulas closed under Adjunction (Adj) and E4-entailment (E4-ent). That is, $a$ is a E4-theory if whenever $A, B \in a$, then $A \wedge B \in a$; and if whenever $A \rightarrow B$ is a theorem of $E_{4}$ and $A \in a, B \in a$. 
Definition 4.2 (Classes of E4-theories). Let a be an E4-theory. We set (1) $a$ is prime iff whenever $A \vee B \in a$, then $A \in a$ or $B \in a$; (2) $a$ is regular iff a contains all theorems of $E 4$.

The standard concept of "set of consequences of a set of wffs" will be useful.

DeFinition 4.3 (The set $\mathrm{Cn} \Gamma[\mathrm{E} 4]$ ). The set of consequences in $E_{4}$ of a set of wffs $\Gamma$ (in symbols $C n \Gamma[E 4]$ ) is defined as follows: $C n \Gamma\left[E_{4}\right]=\{A \mid$ $\left.\Gamma \vdash_{E_{4}} A\right\}$ (cf. Definitions 2.2 and 2.3).

We note the following corollary:

Corollary 4.4 ( $\mathrm{Cn} \Gamma[\mathrm{E} 4]$ is a regular theory). For any $\Gamma, C n \Gamma[E 4]$ is a regular theory.

Proof: It is immediate by Definitions 2.2, 2.3 and 4.3. Clearly, $\mathrm{Cn} \Gamma[\mathrm{E} 4]$ is closed under Adj and MP and contains all theorems of E4. Then, it is closed under E4-ent.

Next, we build the regular prime theory $\mathcal{T}$ upon which the canonical model is defined. But firstly we recall the primeness lemma.

Lemma 4.5 (Extension to prime theories). Let a be a theory and $A$ a wff such that $A \notin a$. Then, there is a prime theory $x$ such that $a \subseteq x$ and $A \notin x$.

Proof: Cf. [6], Chapter 4, where it is shown how to proceed in an ample class of logics including Routley and Meyer's basic positive logic $\mathrm{B}_{+}$by either applying the Extension lemma or Kuratowski-Zorn's lemma (notice that, of course, E4 includes $\left.\mathrm{B}_{+}\right)$.

Proposition 4.6 (The building of $\mathcal{T}$ ). Let $\Gamma$ be a set of wffs and $A$ a wff such that $\Gamma \nvdash_{E 4} A$. Then, there is a regular prime theory $\mathcal{T}$ such that $\Gamma \subseteq \mathcal{T}$ and $A \notin \mathcal{T}$.

Proof: For $\Gamma$ and $A$, suppose $\Gamma \nvdash_{\mathrm{E} 4} A$. Then, $A \notin \mathrm{Cn} \Gamma[\mathrm{E} 4]$. By Corollary $4.4, \mathrm{Cn} \Gamma[\mathrm{E} 4]$ is a regular theory. Hence, by Lemma 4.5, there is a (regular) 
prime theory $\mathcal{T}$ such that $\mathrm{Cn} \Gamma[\mathrm{E} 4] \subseteq \mathcal{T}$ and $A \notin \mathcal{T}$. Thus, $\Gamma \subseteq \mathcal{T}$ and $A \notin \mathcal{T}$, since $\Gamma \subseteq \mathrm{Cn} \Gamma[\mathrm{E} 4]$, as it was required.

The canonical model is defined upon the theory $\mathcal{T}$ just built as follows. In the first place, we define some preliminary notions.

Definition $4.7\left(R^{P}, *^{P}\right.$ and $\left.\models^{P}\right)$. Let $K^{P}$ be the set of all prime theories. Then, $R^{P}, *^{P}$ and $\vDash^{P}$ are defined as follows for all $a, b, c \in K^{P}$ and wffs $A, B$ : (i) $R^{P} a b c$ iff $(A \rightarrow B \in a \& A \in b) \Rightarrow B \in c$; (ii) $a^{*^{P}}=\{A \mid \neg A \notin$ a\}; (iii) $a \vDash^{P} A$ iff $A \in a$.

Now, we show that $*^{P}$ is an operation on $K^{P}$.

Proposition $4.8\left(*^{P}\right.$ is an operation on $\left.K^{P}\right)$. (1) Let a be a prime theory. Then, $a^{*^{P}}$ is a prime theory as well. (2) Moreover, for any $A, \neg A \in a^{*^{P}}$ iff $A \notin a$.

Proof: Cf. [6], Chapter 4. (1) $a^{*^{P}}$ is closed under E4-ent by T6; $a^{*^{P}}$ is closed under Adj by T4; $a^{*^{P}}$ is prime by T5. (2) By T2 and T3.

Notice that $a^{*^{P}}$ is not necessarily regular if $a$ is regular. Also, remark that $\mathcal{T}^{*^{P}}$ is a prime theory. We can now define the canonical model.

Definition 4.9 (The canonical E4-model). The canonical E4-model is the structure $\left(K^{C}, *^{C}, R^{C}, \models^{C}\right)$ where $K^{C}=\left\{\mathcal{T}, \mathcal{T}^{*^{C}}\right\}, \mathcal{T}$ is the regular prime theory built in Proposition 4.6 and $*^{C}, R^{C}$ and $\models^{C}$ are the restrictions of $*^{P}, R^{P}$ and $\vDash^{P}$ to $K^{C}$. (Notice that for any set of wffs $\Gamma$ and wff $A, \Gamma \vDash^{C} A$ iff $\mathcal{T} \vDash^{C} A$ if $\mathcal{T} \vDash^{C} \Gamma\left(\mathcal{T} \models^{C} \Gamma\right.$ iff $\mathcal{T} \vDash^{C} B$ for each $\left.B \in \Gamma\right)$.)

We need to prove that the canonical E4-model is indeed an E4-model. This requires proving a series of preliminary facts. We begin by showing that $*^{P}$ is an involution in $K^{P}$. (In the rest of the section the superscripts $P$ and $C$ are generally omitted above $*$ and $R$ when there is no risk of confusion.)

Proposition $4.10\left(a=a^{* *}\right)$. For any $a \in K^{P}, a=a^{*^{P} *^{P}}$.

Proof: Immediate by T2, T3 and closure of $a$ under E4-ent. 
As a corollary of Proposition 4.10, we have:

Corollary $4.11\left(*^{C}\right.$ is an involutive operation on $\left.K^{C}\right)$. The operation $*^{C}$ is an involutive operation on $K^{C}$. That is, for any $a \in K^{C}, a^{*^{C}} \in K^{C}$ and, moreover, $a=a^{*^{C} *^{C}}$.

Proof: Immediate by Proposition 4.8 and Proposition 4.10 .

Thus, in order to prove that the canonical model is a model, it remains to prove that both the ternary relation $R$ and the (valuation) clauses in Definition 3.1 hold canonically.

LEMma 4.12 ( $R$ holds canonically). If $a, b, c \in K^{C}$, then $R^{C} a b c$ iff $a=\mathcal{T}^{*^{C}}$ or $b=c$.

Proof: Given Corollary 4.11, it suffices to prove that the following relations hold: (a) $R \mathcal{T} \mathcal{T}$; (b) $R \mathcal{T}^{*} \mathcal{T}^{*} \mathcal{T}^{*} ;$ (c) $R \mathcal{T}^{*} \mathcal{T}^{*} \mathcal{T}$; (d) $R \mathcal{T}^{*} \mathcal{T}^{*}$; (e) $R \mathcal{T} \mathcal{T}^{*} \mathcal{T}^{*}$; (f) $R \mathcal{T}^{*} \mathcal{T} \mathcal{T}$.

(a) $R \mathcal{T} \mathcal{T} \mathcal{T}$ : For wffs $A, B$, suppose $A \rightarrow B \in \mathcal{T}$ and $A \in \mathcal{T}$. Then, $B \in \mathcal{T}$ follows immediately by $\mathrm{T} 1([A \wedge(A \rightarrow B)] \rightarrow B)$.

(b) $R \mathcal{T}^{*} \mathcal{T}^{*} \mathcal{T}^{*}$ : For wffs $A, B$, suppose (1) $A \rightarrow B \in \mathcal{T}^{*}$ and $A \in \mathcal{T}^{*}$. Then, $(2) \neg(A \rightarrow B) \notin \mathcal{T}$ and $\neg A \notin \mathcal{T}$. By T7 $(\neg B \rightarrow[\neg A \vee(\neg A \rightarrow B)])$, 2 and the primeness of $\mathcal{T}, \neg B \notin \mathcal{T}$, whence $B \in \mathcal{T}^{*}$, as it was required.

(c) $R \mathcal{T}^{*} \mathcal{T}^{*} \mathcal{T}$ : For wffs $A, B$, suppose (1) $A \rightarrow B \in \mathcal{T}^{*}$ and $A \in \mathcal{T}^{*}$. And, for reductio, $(2) B \notin \mathcal{T}$. We have $(3) \neg B \in \mathcal{T}^{*}$. Now A12 $([(A \rightarrow$ $B) \wedge(A \wedge \neg B)] \rightarrow \neg(A \rightarrow B))$ is applied and we have $(4) \neg(A \rightarrow B) \in \mathcal{T}^{*}$ by 1 and 3 , whence (5) $A \rightarrow B \notin \mathcal{T}$ by Proposition 4.8(2). Next, A13 $((A \rightarrow B) \vee \neg(A \rightarrow B))$ is applied and we get $(6) \neg(A \rightarrow B) \in \mathcal{T}$. Finally, again by Proposition 4.8(2) and 6 , we have (7) $A \rightarrow B \notin \mathcal{T}^{*}$. But 1 and 7 contradict each other.

(d) $R \mathcal{T}^{*} \mathcal{T} \mathcal{T}^{*}$ : The proof is similar to that of case $\mathrm{c}$ by using again A12 and A13.

(e) $R \mathcal{T} \mathcal{T}^{*} \mathcal{T}^{*}$ : For wffs $A, B$, suppose $A \rightarrow B \in \mathcal{T}$ and $A \in \mathcal{T}^{*}$. Then, $\neg A \notin \mathcal{T}$. By T6 $((A \rightarrow B) \rightarrow(\neg B \rightarrow \neg A))$ we have $\neg B \rightarrow \neg A \in \mathcal{T}$ whence $\neg B \notin \mathcal{T}$ and, finally, $B \in \mathcal{T}^{*}$, as it was to be proved.

(f) $R \mathcal{T}^{*} \mathcal{T} \mathcal{T}$ : For wffs $A, B$, suppose (1) $A \rightarrow B \in \mathcal{T}^{*}$ and $A \in \mathcal{T}$. By T8 $(A \rightarrow[B \vee \neg(A \rightarrow B)])$ we have $B \vee \neg(A \rightarrow B) \in \mathcal{T}$. But $\neg(A \rightarrow B) \notin \mathcal{T}$ follows by 1 . So, we have $B \in \mathcal{T}$ by the primeness of $\mathcal{T}$. 
Lemma 4.13 (Clauses (i)-(v) hold canonically). Clauses (i)-(v) in Definition 3.1 are satisfied by the canonical E4-model.

Proof: (Cf. Definitions 4.7, 4.9.) (i) is immediate; (ii) follows by A2 and closure of theories under Adj; (iii) is proved by A4 and primeness of $\mathcal{T}$ and $\mathcal{T}^{*}$; and (v) and (iv) (from left to right) are immediate by Definition 4.9. So, let us prove (iv) from right to left.

(I) Suppose $A \rightarrow B \notin \mathcal{T}$ for wffs $A, B$. We have to prove $(R \mathcal{T} \mathcal{T} \mathcal{T} \&$ $A \in \mathcal{T} \quad \& \quad B \notin \mathcal{T})$ or $\left(R \mathcal{T} \mathcal{T}^{*} \mathcal{T}^{*} \& A \in \mathcal{T}^{*} \& B \notin \mathcal{T}^{*}\right)$. Suppose, for reductio, that this is not the case. Then, at least one of the following four alternatives is obtained: (i) $A \notin \mathcal{T} \& A \notin \mathcal{T}^{*}$; (ii) $A \notin \mathcal{T} \& B \in \mathcal{T}^{*}$; (iii) $B \in \mathcal{T} \quad \& \quad A \notin \mathcal{T}^{*}$ and (iv) $B \in \mathcal{T} \quad \& \quad B \in \mathcal{T}^{*}$. We show that each one of these four alternatives is untenable thus proving case I. Firstly, notice that, given (a) $A \rightarrow B \notin \mathcal{T}$, by A13 $((A \rightarrow B) \vee \neg(A \rightarrow B))$, we have (b) $\neg(A \rightarrow B) \in \mathcal{T}$. Next, we examine, i-iv.

(i) $A \notin \mathcal{T} \& A \notin \mathcal{T}^{*}$ : Then $(1) \neg A \in \mathcal{T}$. By T9 $([\neg(A \rightarrow B) \wedge \neg A] \rightarrow$ $A)$, b and 1, (2) $A \in \mathcal{T}$. But 2 contradicts i.

(ii) $A \notin \mathcal{T} \& B \in \mathcal{T}^{*}$ : Then $(1) \neg B \notin \mathcal{T}$. By T10, $(A \vee \neg B) \vee(A \rightarrow$ $B) \in \mathcal{T}$. Thus, (2) $A \in \mathcal{T}$ by a and 1 . But 2 contradicts ii.

(iii) $B \in \mathcal{T} \quad \& \quad A \notin \mathcal{T}^{*}$ : Then (1) $\neg A \in \mathcal{T}$. By A14, $([\neg(A \rightarrow$ $B) \wedge(\neg A \wedge B)] \rightarrow(A \rightarrow B))$, b, iii and 1 , we have $(2) A \rightarrow B \in \mathcal{T}$. But 2 contradicts a.

(iv) $B \in \mathcal{T} \quad \& \quad B \in \mathcal{T}^{*}$ : Then $(1) \neg B \notin \mathcal{T}$. By T11, $([\neg(A \rightarrow$ $B) \wedge B] \rightarrow \neg B$ ), b, and iv, we get $(2) \neg B \in \mathcal{T}$. But 2 contradicts 1 .

(II) Suppose $A \rightarrow B \notin \mathcal{T}^{*}$ for wffs $A, B$. Given $R \mathcal{T}^{*} \mathcal{T}^{*} \mathcal{T}^{*}, R \mathcal{T}^{*} \mathcal{T}^{*} \mathcal{T}$, $R \mathcal{T}^{*} \mathcal{T} \mathcal{T}^{*}$ and $R \mathcal{T}^{*} \mathcal{T} \mathcal{T}$, we have to prove: $\left(A \in \mathcal{T}^{*} \& B \notin \mathcal{T}^{*}\right)$ or $\left(A \in \mathcal{T}^{*}\right.$ $\& B \notin \mathcal{T})$ or $\left(A \in \mathcal{T} \quad \& \quad B \notin \mathcal{T}^{*}\right)$ or $(A \in \mathcal{T} \quad \& \quad B \notin \mathcal{T})$. For reductio suppose that this is not the case. We then are given 16 alternatives. But it is easy to check that in each one of these 16 alternatives we have (i) $A \notin \mathcal{T} \& A \notin \mathcal{T}^{*}$ and/or (ii) $B \in \mathcal{T} \& B \in \mathcal{T}^{*}$. But i and ii are proved untenable as in subcases $\mathrm{i}$ and iv of case $\mathrm{I}$.

We can now prove completeness, once shown that the canonical E4model is an E4-model.

LEMma 4.14 (The canonical E4-model is an E4-model). The canonical E4model is indeed an E4-model. 
Proof: By Corollary 4.11, $*^{C}$ is an involutive operation on $K^{C}$; by Lemma 4.12 , the ternary relation $R^{C}$ holds canonically, and, finally, by Lemma 4.13 , the (valuation) classes $\mathrm{i}-\mathrm{v}$ hold when interpreted canonically.

Theorem 4.15 (Completeness of E4). For any set of wffs $\Gamma$ and wff $A$, if $\Gamma \vDash_{E_{4}} A$, then $\Gamma \vdash_{E_{4}} A$.

Proof: Suppose $\Gamma \nvdash_{\mathrm{E} 4} A$ for $\Gamma$ and $A$. By Proposition 4.6, there is a regular prime theory $\mathcal{T}$ such that $\Gamma \subseteq \mathcal{T}$ and $A \notin \mathcal{T}$. Then, the canonical E4-model is defined upon $\mathcal{T}$ as shown in Definition 4.9. By Lemma 4.14, the canonical E4-model is an E4-model. Then, $\Gamma \not \nvdash^{C} A$ since $\mathcal{T} \models^{C} \Gamma$ but $\mathcal{T} \not \nvdash^{C} A$. Thus, $\Gamma \not \nvdash_{\mathrm{E} 4} A$ by Definition 3.4.

\section{The necessity operator}

In [5], it is shown that a necessity operator can be introduced in E4 via the definition $\square A=_{d f}(A \rightarrow A) \rightarrow A$, similarly as in Anderson and Belnap's logic of entailment (cf. [1], $\S 4.3$ and references therein; the possibility operator is defined in the customary way: $\diamond A=_{d f} \neg \square \neg A$ ). The resulting truth tables are (cf. section 2):

\begin{tabular}{l|llll} 
& 0 & 1 & 2 & 3 \\
\hline$\square$ & 0 & 0 & 2 & 3
\end{tabular}

\begin{tabular}{l|llll} 
& 0 & 1 & 2 & 3 \\
\hline$\diamond$ & 0 & 3 & 2 & 3
\end{tabular}

Then $\square$ could be interpreted in E4-models by introducing a binary accessibility relation in the usual way. This relation $S$ is reflexive (for all $x \in K, S x x)$ and, in addition, $S 0^{*} 0$ holds but $S 00^{*}$ does not. The clause for $\square$ is , of course:

(vi). For all $a \in K, a \vDash \square A$ iff for all $b \in K, S a b \Rightarrow b \vDash A$

We prove:

Proposition $5.1\left(\vDash_{\mathrm{E} 4}(A \rightarrow A) \rightarrow A\right.$ iff $\left.S 0 x \Rightarrow x \vDash A\right)$. For any E4-model and wff $A, 0 \vDash(A \rightarrow A) \rightarrow A$ iff for any $x \in K$, if $S 0 x$, then $x \vDash A$.

Proof: Let $\mathrm{M}$ be any E4-model and $A$ be a wff. Subcase (i). Suppose (1) $0 \vDash(A \rightarrow A) \rightarrow A$ and (2) $S 0 x$, for any $x \in K$. We have to consider only the case (3) $S 00$. (Recall that $S 00^{*}$ does not hold.) By Remark 3.6(a), (4) $R 000$; on the other hand we obviously have (5) $0 \vDash A \rightarrow A$. Then, 
(6) $0 \vDash A$ follows (by 1, 4 and 5), as it was required. Subcase (ii). Suppose (1) $S 0 x \Rightarrow x \vDash A$ (for any $x \in K$ ) and (2) $x \vDash A \rightarrow A$ (for any $x \in K$ ). We have to prove $x \vDash A$. By reflexivity of $S$, (3) $S 00$. Then, (4) $0 \vDash A$ follows by 1 and 3 . So, if $x$ is 0 , subcase ii is proved. Let then $x$ be $0^{*}$. By Remark $3.6(\mathrm{~d}),(5) R 0^{*} 00^{*}$. Thus, we get (6) $0^{*} \vDash A$ by 2,4 and 5 .

This necessity operator could be introduced (independently of the definition via $\rightarrow$ ) by means of the following axioms and rules:

A16. $\square A \rightarrow A$

A17. $(\square A \wedge \neg \square A) \rightarrow \neg A$

A18. $A \vee \neg \square A$

\section{Necessitation (Nec). $A \Rightarrow \square A$ \\ Disjunctive Necessitation (dNec). $B \vee A \Rightarrow B \vee \square A$}

Given soundness and completeness of E4, we sketch a proof of the soundness and completeness of E4 plus axioms A16, A17, A18, Nec and dNec. (Let us refer by $\mathrm{E} 4 \square$ to this expansion of E4.)

Theorem 5.2 (Soundness of E4 $\square$ ). For any set of wffs $\Gamma$ and wff $A$, if $\Gamma \vdash_{E 4 \square} A$, then $\Gamma \vDash_{E 4 \square} A$.

Proof: (Sketch) $A 16 \square A \rightarrow A$ is E4 $\square$-valid: Case (i) $0 \vDash \square A \Rightarrow 0 \vDash A$ and case (ii) $0^{*} \vDash \square A \Rightarrow 0^{*} \vDash A$ are immediate by reflexivity of $S$.

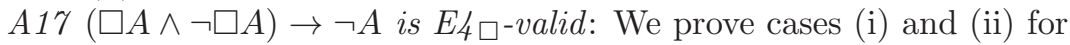
an arbitrary E4-model. Case (i): $0 \vDash \square A \wedge \neg \square A \Rightarrow 0 \vDash \neg A$. Suppose (1) $0 \vDash \square A, 0 \vDash \neg \square A$ (i.e., $0^{*} \not \models \square A$ ) and (2) $0 \not \models \neg A$ (i.e., $0^{*} \vDash A$ ). By $1\left(0^{*} \not \models \square A\right.$ ), we have (3) $S 0^{*} x$ and $x \not \models A$ for some $x \in K$. Now, $x=0^{*}$ is impossible since $0^{*} \vDash A$ by 2 . So, let us suppose (4) $x=0$. Then (5) $S 0^{*} 0$ and $0 \not \models A$ by 3. But, by $1(0 \vDash \square A)$ and reflexivity of $S$, (6) $0 \vDash A$, contradicting 5 .

Case (ii): $0^{*} \vDash \square A \wedge \neg \square A \Rightarrow 0^{*} \vDash \neg A$. Suppose (1) $0^{*} \vDash \square A, 0^{*} \vDash \neg \square A$ (i.e., $0 \not \models \square A$ ) and (2) $0^{*} \not \models \neg A$ (i.e., $0 \vDash A$ ). By $1(0 \not \models \square A$ ), we have (3) $S 0 x$ and $x \not \models A$ for some $x \in K$. But the only possibility is $S 00$. Then, $0 \not \models A$, by 3 , contradicting 2

$A 18 A \vee \neg \square A$ is $E 4 \square$-valid: Suppose (1) $0 \not \models A$ and (2) $0 \not \models \neg \square A$ (i.e., $0^{*} \vDash \square A$ ). Given $S 0^{*} 0$, we have (3) $0 \vDash A$, contradicting 1 . 
Finally, Nec and dNec are immediate by reflexivity of $S$.

In what follows we turn to completeness.

Theorem 5.3 (Completeness of E4 $\square$ ). For any set of wffs $\Gamma$ and wff $A$, if $\Gamma \vDash_{E 4 \square} A$, then $\Gamma \vdash_{E 4 \square} A$.

PROOF: (Sketch) In the first place, the notion of an E4 $\square$-theory is introduced. An E4 $\square$-theory $a$ is, as an E4-theory (cf. Definition 4.1), a set of wffs closed under Adj and $\mathrm{E} 4 \square$-entailment. But, in addition, $a$ is also closed under Nec and dNec. That is, for wffs $A, B$, (i) if $A \in a$, then $\square A \in a$; and (ii) if $B \vee A \in a$, then $B \vee \square A \in a$. Then, dNec is essentially used in the extension to prime theories lemma (Lemma 4.5) in order to build a regular prime theory closed under Nec and dNec (consult [6], Chapter 4, pp. 336, $\mathrm{ff}$. on the use of disjunctive rules in building prime theories defined upon logics closed under weak rules of derivation).

Next, we prove that the relation $S$ and clause vi hold when interpreted canonically, i.e., $a \vDash^{C} \square A$ (for all $a \in K^{C}$ and wff $A$ ) is interpreted according to Definition 4.9, and $S^{C} a b$ (for all $a, b \in K^{C}$ ) is interpreted as follows: $S^{C} a b$ iff for any wff $A, \square A \in a \Rightarrow A \in b$.

(1) $S^{C} \mathcal{T} \mathcal{T}$ and $S^{C} \mathcal{T}^{*} \mathcal{T}^{*}$ are immediate by A16.

(2) $S^{C} \mathcal{T}^{*} \mathcal{T}$ : Suppose $\square A \in \mathcal{T}^{*}$ for wff $A$. Then, $\neg \square A \notin \mathcal{T}$ and next $A \in \mathcal{T}$ follows by A18 and primeness of $\mathcal{T}$.

(3) For all $a \in K^{C}, a \vDash^{C} \square A$ iff for all $b \in K^{C}, S^{C} a b \Rightarrow b \vDash^{C} A$. The canonical clause vi is immediate from left to right. So, let us prove it from right to left.

(I) Suppose $\square A \notin \mathcal{T}$ for wff $A$. We have to prove $S \mathcal{T} \mathcal{T} \& A \notin \mathcal{T}$. But this follows by closure of $\mathcal{T}$ under Nec, since if $A \in \mathcal{T}$, then $\square A \in \mathcal{T}$.

(II) Suppose (1) $\square A \notin \mathcal{T}^{*}$ for wff $A$. We have to prove $\left(S \mathcal{T}^{*} \mathcal{T}^{*} \quad \&\right.$ $\left.A \notin \mathcal{T}^{*}\right)$ or $\left(S \mathcal{T}^{*} \mathcal{T} \& A \notin \mathcal{T}\right)$. For reductio, suppose $(2) A \in \mathcal{T}^{*}$ (i.e., $\neg A \notin \mathcal{T})$ and (3) $A \in \mathcal{T}$. Now, $A \rightarrow(\neg A \vee \square A)$ is a theorem of E4 $\square$. Then, by 2, 3 and this theorem we have (using in addition the primeness of $\mathcal{T}$ ), (4) $\square A \in \mathcal{T}$. By 1, (5) $\neg \square A \in \mathcal{T}$ follows. But by 4,5 and A17 $((\square A \wedge \neg \square A) \rightarrow \neg A$ ), we get $(6) \neg A \in \mathcal{T}$, contradicting 2 .

ACKNOWLEDGEMENTs. Work supported by research project FFI201453919-P financed by the Spanish Ministry of Economy and Competitiveness. We thank two referees of the BSL whose remarks and suggestions lead 
to an improved version of a first version of the present paper. This paper was written following a suggestion by John Slaney (cf. [5], §9), which, of course, is not to blame by how we interpreted his suggestion or by any errors or infelicities that the preceding pages may contain.

\section{References}

[1] A. R. Anderson, N. D. Belnap, Entailment. The Logic of Relevance and Necessity, vol. I, Princeton University Press (1975).

[2] R. T. Brady, Completeness Proofs for the Systems RM3 and BN4, Logique et Analyse 25 (1982), pp. 9-32.

[3] C. González, MaTest. Available at: http://ceguel.es/matest (2012). Last accessed: 23/03/2016.

[4] R. K. Meyer, S. Giambrone, R. T. Brady, Where gamma fails, Studia Logica 43 (1984), pp. 247-256.

[5] G. Robles, J. M. Méndez (2016), A companion to Brady's 4-valued relevant logic BN4: The 4-valued logic of entailment E4, Logic Journal of the IGPL 24(5), pp. 838-858, doi: 10.1093/jigpal/jzw011.

[6] R. Routley, R. K. Meyer, V. Plumwood, R. T. Brady (1982), Relevant Logics and their Rivals, vol. 1, Ridgeview Publishing Co., Atascadero (1982).

G. Robles

Dpto. de Psicología, Sociología y Filosofía, Universidad de León

Campus de Vegazana, s/n, 24071, León, Spain

http://grobv.unileon.es

e-mail: gemma.robles@unileon.es

S. M. López*, J. M. Blanco*, M. M. Recio**, J. R. Paradela* Dpto. de Filosofía, Lógica y Estética, Universidad de Salamanca Campus Unamuno, Edificio FES, 37007, Salamanca, Spain e-mail: u127548@usal.es; jmblanco@usal.es; marcosmanuelrecioperez@usal.es; jrparadela@hotmail.es

*: Graduate student

**: Undergraduate student 